\title{
IMPEDANCE MEASUREMENT IN A HYDROSTATIC DRIVE
}

\author{
Benedikt Müller $^{1 *}$, Heiko Baum ${ }^{1}$ \\ ${ }^{1}$ FLUIDON Gesellschaft für Fluidtechnik mbH, Jülicher Straße 338a, 52070 Aachen \\ * Corresponding author: Tel.: +49 2419609 260; E-mail address: benedikt.mueller@fluidon.com
}

\begin{abstract}
Pressure oscillation in hydrostatic drive trains can cause noise and damage to components. They impair function and reliability. The visualization of the oscillation mode helps to clarify the causal relationships in the hydrostatic drive train and is a basis for the development of remedial measures.

Analysis of the pressure oscillation situation, however, can only be carried out in the complete system, since line branching and the impedance of the hydrostats have an influence on the resonance frequencies and the oscillation modes. If only the line length between the components is considered in the pressure oscillation analysis, neither the calculated frequencies nor the position of the pressure antinodes where possible remedial measures are to be placed are correct.

This paper presents the metrological determination of the impedance of a hydrostat on a functional test bench ("mobile impedance measurement") and the preparation of the measurement data for the subsequent simulative pressure oscillation analysis of a hydraulic drive train.
\end{abstract}

Keywords: Noise, Vibration, Pressure Oscillation, Pulsation, Impedance, Hydrostatic Drive

\section{INTRODUCTION}

In the case of commercial vehicles, the focus of development is on fulfilling the intended function. The hydrostatic drive train (Figure 1) as a subsystem of the vehicle concept is therefore subject to design restrictions regarding component positioning or line routing.

The required flexibility in the layout of the drive train repeatedly challenges the developers regarding the pressure oscillation behavior of the lines. The entire powertrain is excited by pressure pulsations in a wide frequency spectrum by the hydrostats' pulsations, and it must be ensured that no disturbing resonance oscillation occur. With modern fluidic 1D simulation tools [[2], it is now possible to predict under which operating conditions (steady-state pressure, dynamic pressure amplitude, frequency of pulsation) the drive train is subject to pressure oscillations. However, the acoustic behavior of all components installed in the drive train must be known, which is typically not the case for the hydrostatic displacement units in particular.

\subsection{Pressure oscillation analysis}

In principle, every hydraulic system, including a hydrostatic drive train, has several natural frequencies [3]. However, this does not necessarily

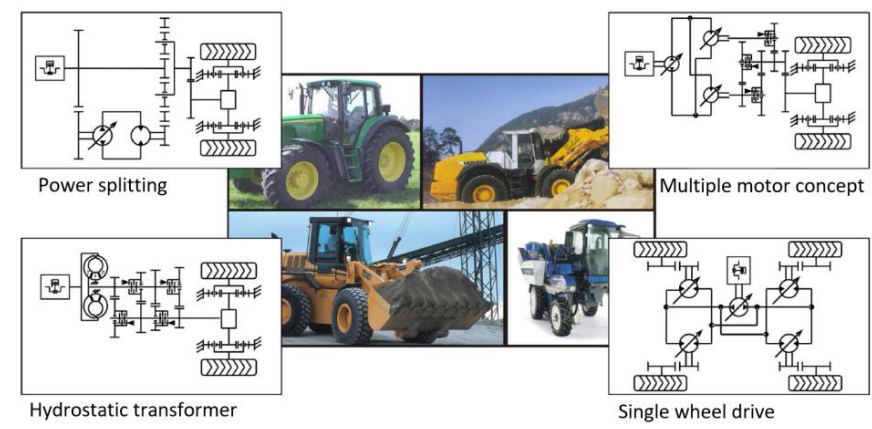

Figure 1: Schematics and application examples of different drive train concepts [1] 
mean that resonance problems occur in the system. For this purpose, the natural frequencies must first be excited ("hit"). An excitation inevitably occurs in the drive train due to the reversal processes and kinematic irregularities of the hydrostats, which cause volume flow pulsations. Flow pulsations and resulting pressure pulsations travel as waves through the entire hydraulic system. Due to the variable speed of the hydrostats, the hydraulic system is excited by the pressure pulsations in a wide frequency spectrum, which increases the probability that a system resonance is "hit".

If a countermeasure is to be designed for a pressure oscillation problem in the drive train, it is not enough to identify the natural frequencies and the pressure pulsation amplitudes by means of measurements. As the following introduction to simulative pressure oscillation analysis shows, a deeper understanding of the interactions between the individual subsystems is essential. The example of a straight pipe of length $L_{P}$, closed on both sides, with constant diameter $d_{P}$ (Figure 2) is the first step into pressure oscillation analysis.

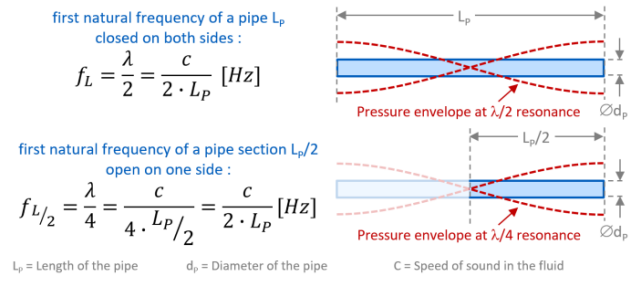

Figure 2: First natural frequency of a pipeline closed on both sides

Pressure and flow pulsations propagate as plane waves in the pipe and are reflected at the pipe ends. The dynamic behavior of the line terminating elements, the so-called terminating impedance, has a decisive influence on how the pressure waves are reflected. For additional information on sound processes in pipes, please refer to [4] and for technical acoustics in general to [5].

The closed end of a straight pipe represents the special case of an infinite terminating impedance, resulting in the reflection of a pressure wave with the reflection factor $R=1$ (total reflection). The first natural frequency of the pipe is calculated using the formula shown on top of Figure 2, which shows that a pipe closed at both ends is subject to the $\lambda / 2$ resonance condition.

Figure 2 also depicts the envelope of alternat- ing pressure in the $\lambda / 2$ resonance case. The envelope is symmetrical, and the pressure node is exactly in the middle of the pipe. If the pipe is cut exactly in the pressure node, then two subsystems with the length $L_{P} / 2$ are created, which are now open on one side. The terminating impedance of an open line termination is zero, which is again a special case. A pressure wave is reflected at the open pipe end with the reflection factor $R=-1$. The first natural frequency of the unilaterally open pipe section is calculated using the formula shown in the lower part of Figure 2. The example shows that a unilaterally open pipe section is subject to the $\lambda / 4$ resonance condition. The first natural frequency $\left(\lambda / 2\right.$-resonance) of the pipe $L_{P}$, which is closed on both ends, is thus composed of two subsystems which are subject to the $\lambda / 4$-resonance condition and which abut with their open ends.

Figure 3 shows the effect that occurs in the pipe when the excitation frequency "hits" the natural frequency. The displayed "pressure vector plot" is a color scaled top view of the pressure values stored during the simulation. An explanation of the simulative procedure is given in [6].

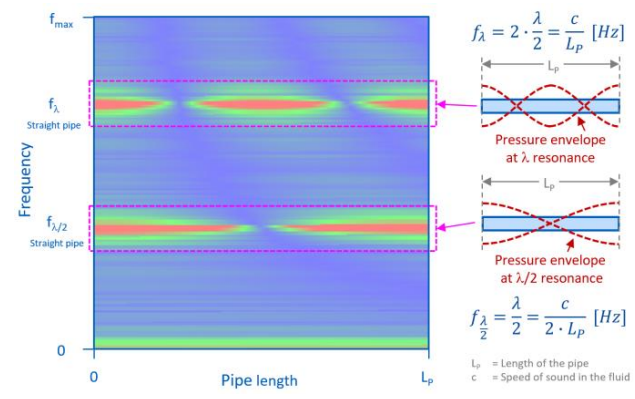

Figure 3: Pressure vector plot of the straight pipe closed on both sides

The $x$-axis starts at the pulsation source (e.g. the pump), which also defines the origin $x=0$ of the pipe and ends at $x=L_{P}$ at a load. The y-axis does not show the simulation time, but the frequency of the excitation. The excitation frequency can also be converted into a hydrostatic speed depending on the number of displacers (number of pistons, blades or teeth). The horizontal lines indicate the first natural frequency $(\lambda / 2$-resonance) and the second natural frequency ( $\lambda$-resonance).

The pressure vector plot in Figure 3 clearly shows when the excitation frequency "hits" the natural frequencies of the pipe at $f_{\lambda / 2}$ and at $f_{\lambda}$. If 
the excitation frequency is continuously increased, the resonances initially build up slowly, reach their maxima and decay again. In top view, the shape of the visualized pressure pulsation resembles the envelope shown in Figure 2, making the resonances very easy to identify. A symmetrical distribution can also be seen at the second natural frequency of the pipe. Here the same decomposition approach (Figure 4) as for the first natural frequency in Figure 2 can be applied by cutting in the pressure nodes.

$$
\begin{gathered}
\text { second natural frequency of a } \\
\text { pipe } L_{p} \text { closed on both sides: } \\
f_{L}=2 \cdot \frac{\lambda}{2}=\frac{c}{L_{P}}[\mathrm{~Hz}] \\
\text { first natural frequency of a } \\
\text { pipe section } L_{p} / 4 \text { open on one side: } \\
f_{L / 4}=\frac{\lambda}{4}=\frac{c}{4 \cdot L_{P} / 4}=\frac{c}{L_{P}}[\mathrm{~Hz}] \\
\text { first natural frequency of a pipe } \\
\text { section } L_{p} / 2 \text { open on both sides: } \\
f_{L_{/}}=\frac{\lambda}{2}=\frac{\lambda}{2 \cdot L_{P} / 2}=\frac{c}{L_{P}}[\mathrm{~Hz}]
\end{gathered}
$$
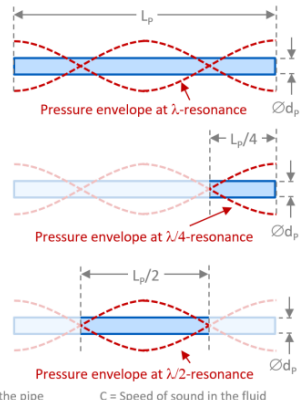

Figure 4: Second natural frequency of a pipe closed on both sides

Three subsystems are created, whose first natural frequency is equal to the frequency of the currently considered natural frequency order of the entire system. Figure 4 also shows that a pipe open on both ends is also subject to the $\lambda / 2$ resonance condition, except that here the pressure antinode of the first natural frequency is situated at the center of the pipeline.

\subsection{Consideration of hydrostats}

A hydrostat acoustically represents a closed line termination. However, before the pressure waves are reflected inside the hydrostat, they must pass through a sequence of channels and volumes. The theoretical analysis of the pressure oscillations in a hydrostat is therefore difficult and can only be carried out with great effort using physical simulation models. Therefore, in pressure oscillation analysis the hydrostats are usually described by their so-called impedance.

For the pressure oscillation analysis, it is only relevant that a pressure wave coming from the pipe enters the hydrostat, somehow gets out of the hydrostat at some point and is superimposed on the pressure wave entering the hydrostat at that point. Depending on the phase difference be- tween the two pressure waves, the pressure amplitude is then increased $(\Delta \varphi=0)$ or reduced $\left(\Delta \varphi=180^{\circ}\right)$. The parameter that describes this behaviour is the impedance of the hydrostat.

The impedance is a complex frequency-dependent quantity that can be determined by measurement. In the next section, the standard measurement methods are explained with their advantages and disadvantages, and then the measurement method in the hydrostatic circuit presented in this article is discussed.

Once the impedance has been determined metrologically, the reproduction of the impedance curve by means of an analogous model is an elegant solution to incorporate it in the simulation. The hybrid pump model presented in [7] is available for the simulative pressure oscillation analysis.

One of the simplest replacement models of a hydrostat's impedance is based on the analogy between the measured impedance curve and the impedance curve of a Helmholtz resonator. Figure 5 shows the principle schematic of the resonator and the most general formula for calculating its natural frequency, which is identical to the frequency of the attenuation point in the impedance curve of the resonator.

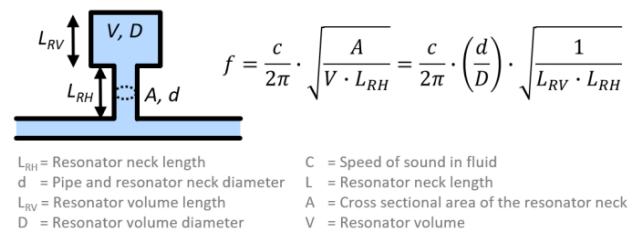

Figure 5: Schematic diagram and natural frequency of a Helmholtz resonator

The impedance curve of the Helmholtz resonator is adapted to the measured impedance of the hydrostat by selecting the parameters volume $V$, cross-sectional area of the resonator neck $A$ and length of the resonator neck $L_{R H}$ accordingly. This is done in the frequency domain and can be automated. Figure 5 does not show the calculation of the speed of sound $c$, considering the pressure-dependent compression modulus of the fluid and the friction in the resonator neck. The characteristic of the attenuation area of the analogous model is adapted to the measurement by the friction. The geometric values are then transferred to the hybrid pump model of the time domain simulation. 
If the example pipe from Figure 2 is now extended by the analogous models of the impedances of two hydrostats, the resulting simulation model corresponds to a simple hydraulic drive train with one pump and one motor. As the pressure vector plot in Figure 6 shows, this system also has its first oscillation mode at $\lambda / 2$.

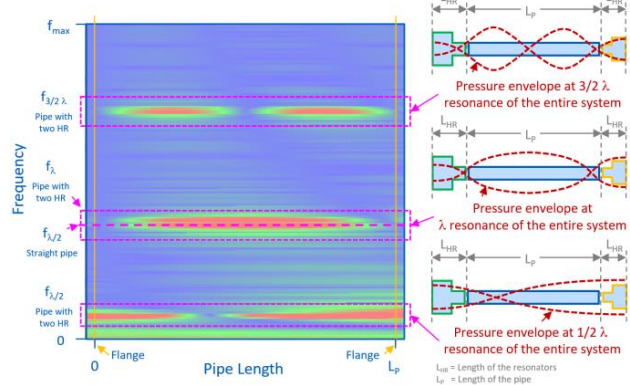

Figure 6: Schematic diagram of the oscillation modes in a hydrostatic transmission

As a result, the highest alternating pressure amplitudes occur at the hydrostats in the resonance case, which then lead to undesirable mechanical loads on sliding components subjected to pressure or on the seals, for example.

\section{TEST PROCEDURE}

Two methods for the experimental determination of the impedance of pumps are described in international standards: The secondary source method (ISO 10767-1:1996 [8]) and the 2 pressures / 2 systems method (2p2s, ISO 10767-1:2015 [9]). Several authors have proposed the use of a third pressure sensor in the measuring section and corresponding evaluation methods to improve the 2p2s method, e.g. [10]. Both methods are briefly presented in the following sections.

Theoretical approaches to determine the pump impedance via geometry and material properties have not yet been successfully applied.

\subsection{Secondary source method}

Figure 7 shows the basic measurement setup for the secondary source method: The test specimen is subjected to pressure pulsations which are generated by a second pulsation source, the "secondary source". These fluid-borne sound waves are reflected in the test specimen and superimpose themselves on the advancing waves.

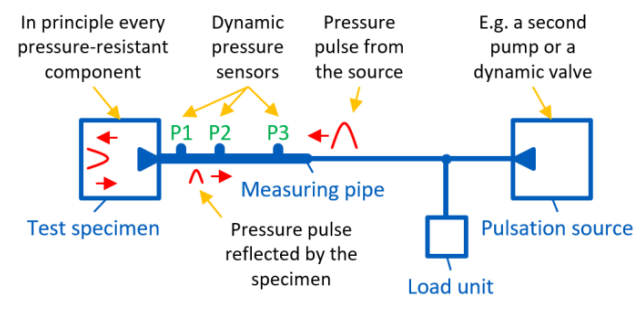

Figure 7: Principle setup of a secondary source test rig [8]

The waves travel through the measuring or reference pipe, which is connected to the pressure port of the test specimen with minimized diameter difference. Dynamic pressure sensors are mounted to this reference pipe to measure the superposition of the forward and backward travelling fluidborne sound waves. If the transmission behaviour of the measuring pipe is known, the impedance of the test specimen can be calculated from the pressure signals. For details please refer to the standard. There you will also find further information on the design of the measuring pipe and recommendations on the sensor distances.

The transmission behaviour of the measuring pipe can be determined the more accurately the more precisely the speed of sound in the measuring pipe is known. It is therefore of decisive importance for the quality of the results. In principle, two pressure sensors in the measuring pipe provide sufficient information to calculate the impedance. However, the use of three sensors offers the essential advantage that the speed of sound can be determined from the same measurement data and thus a significant uncertainty factor in the evaluation can be excluded. The procedure is described in ISO 15086-2:2000 [11].

The test specimen is described as a positive displacement pump in ISO 10767-1:1996. However, the method can be applied to any pressureresistant component. In a variation of the method, passive components (cf. ISO 15086-3:2008 [12]) or motors can also be tested if the pulsation source simultaneously provides the necessary pressure build-up.

\subsection{3p2s method}

The $3 \mathrm{p} 2 \mathrm{~s}$ method ( 3 pressures / 2 systems) is an extension of the $2 \mathrm{p} 2 \mathrm{~s}$ method described in ISO 10767-1:2015. Figure 8 shows the measurement setup based on the standard. Here, the pulsation excitation is performed by the component under 
test itself. The fluid-borne sound waves emitted by the test specimen are reflected at load valve 1 in a first system configuration and at load valve 2 in the second system configuration. The forward and backward travelling waves form a superposition state between the test specimen and the load valve, which is detected by the dynamic pressure sensors in the reference pipe. The impedance is then determined from the measured data of the two system states.

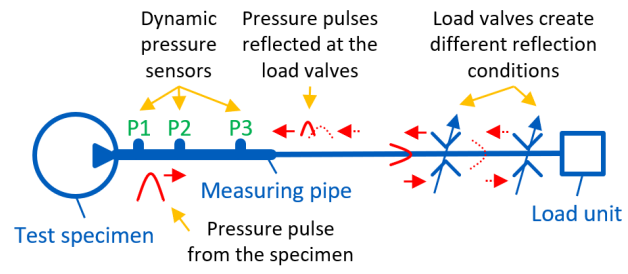

Figure 8: Principle setup of a $3 \mathrm{p} 2 \mathrm{~s}$ test rig $[9,10]$

This method also requires precise knowledge of the transmission behaviour of the measuring section. The use of a third dynamic pressure sensor in the reference section is suggested, for example, in [12], which also allows the speed of sound in the reference pipe to be determined. Furthermore, the three sensors offer the advantage of a possible averaging or the selection of the "suitable" sensor pair (or sensor distance) for a frequency range.

\subsection{Pros and cons}

Both methods have the disadvantage that a special test setup is required, which is particularly costly for larger displacement units. In addition to the drive of the DUT, special attention must be paid to the load unit: This should not contain any components capable of vibrating, as these can lead to disturbance excitations and thus falsify the measurement results. The standards therefore recommend the use of needle valves. With the secondary source method, additional effort is required due to the required pulsation source (secondary source). This can be a servo valve, a second pump or a continuously rotating rotary valve, for example. The use of a pump has the great advantage that motors or passive components can also be tested.

The $2 \mathrm{p} 2 \mathrm{~s}$ or $3 \mathrm{p} 2 \mathrm{~s}$ method avoids the expense of an additional pulsation source, as the test object itself provides the excitation. However, this has the serious disadvantage that without an additional pressure source no motors and, due to the principle, no passive components can be measured. The impedance calculation requires measurement data of two different system states. Compared to the secondary source method, this results in twice the number of test runs and the risk of deviating measurement conditions. The measurement results of the speed of sound shown below indicate that, e.g. different test temperatures can already lead to significantly different measurement results. If the test constellation even requires a conversion from system 1 to system 2 , the potential of deviating test conditions (e.g. air content) and the measuring effort will increase considerably.

The standard includes a stepwise frequency variable excitation by the secondary source. However, the excitation frequencies must not coincide with the pulsation frequencies of the DUT in order to ensure an interference-free measurement. It has proven to be a convenient method in practice to continuously adjust the excitation frequency of the pulsation source ("sweep") and thus cover the entire desired frequency range in one measurement run.

\subsection{Method for impedance measurement in the hydrostatic circuit}

The complex and dedicated test setup, as described in the previous sections, is one of the reasons why the described methods have so far not been widely used in practice for larger displacement units. FLUIDON has taken up the challenge to minimize this effort and to be able to measure pump and motor impedances with a single test setup.

The main requirement is to measure the impedances of the displacement units of a hydrostatic drive on an (existing) drive test bench or even in the machine. The measurement shall be performed in a run-up to continuously obtain data in the whole frequency range of interest.

\section{Separation of desired signal components}

The pressure at a measuring point in a hydrostatic circuit represents an initially uninterpretable mix of pump and motor pulsations that travel back and forth through the system and are partially reflected at various discontinuities. To extract evaluable signals from this "wave salad", the idea was developed to first extract the individual pump and motor orders from the overall signal.

Looking at the pressure signals in the frequency 
range, it becomes obvious that the dominant pump and motor orders can be separated if they have sufficiently different frequencies. The respective frequencies result as a product of the speed, the number of displacers and the order. This relationship is shown in Figure 9 for a runup $n_{\min } \rightarrow n_{\max }$. The transmission ratio of the hydrostatic drive is approximately constant at constant displacements, i.e. there is a fixed speed ratio between pump and motor and the orders of different frequencies can be "separated" over the entire speed range.

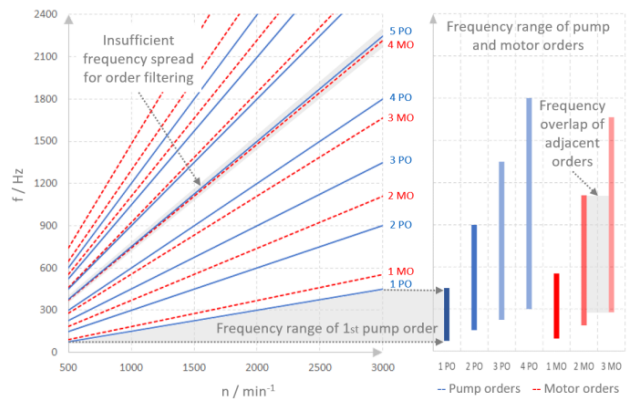

Figure 9: Frequency curves and ranges of the frequency orders of pump and motor during run-up

By varying the transmission ratio, i.e. by changing the pump and/or motor swivel, the speeds can be adjusted so that certain orders are "separable". In the example, the transmission ratio is selected so that the first to fourth pump order and the first to third motor order do not interfere. On the other hand, the frequencies of the $5^{\text {th }}$ pump order and the $4^{\text {th }}$ motor order, for example, are approximately congruent, so that no differentiation in the overall signal would be possible. Each order covers a certain frequency range, depending on the speed range. These are shown in the right diagram in Figure 9. The signals of several orders are available for further calculations in the overlapping ranges.

The extraction of the usable orders from the total signal is done in the time domain by a Butterworth bandpass filter of higher order, whose center frequency is guided by the speed signal.

\section{Development of the method by virtual testing}

The methodology of the "mobile impedance measurement" was developed in a virtual test using 1D simulation. This made it possible to prove the feasibility and test the method before the hardware was available. Expensive test bench time could be saved, and the subsequent measurement campaign could be carried out much more efficiently.

Figure 10 shows the simplified structure of the simulation model, which depicts the highpressure part of the hydrostatic circuit. The displacement units are shown in the model as a combination of a volume flow source or sink and a branch resonator and describe the hybrid pump model presented in [7]. The branch resonator represents the impedance of a displacement unit in

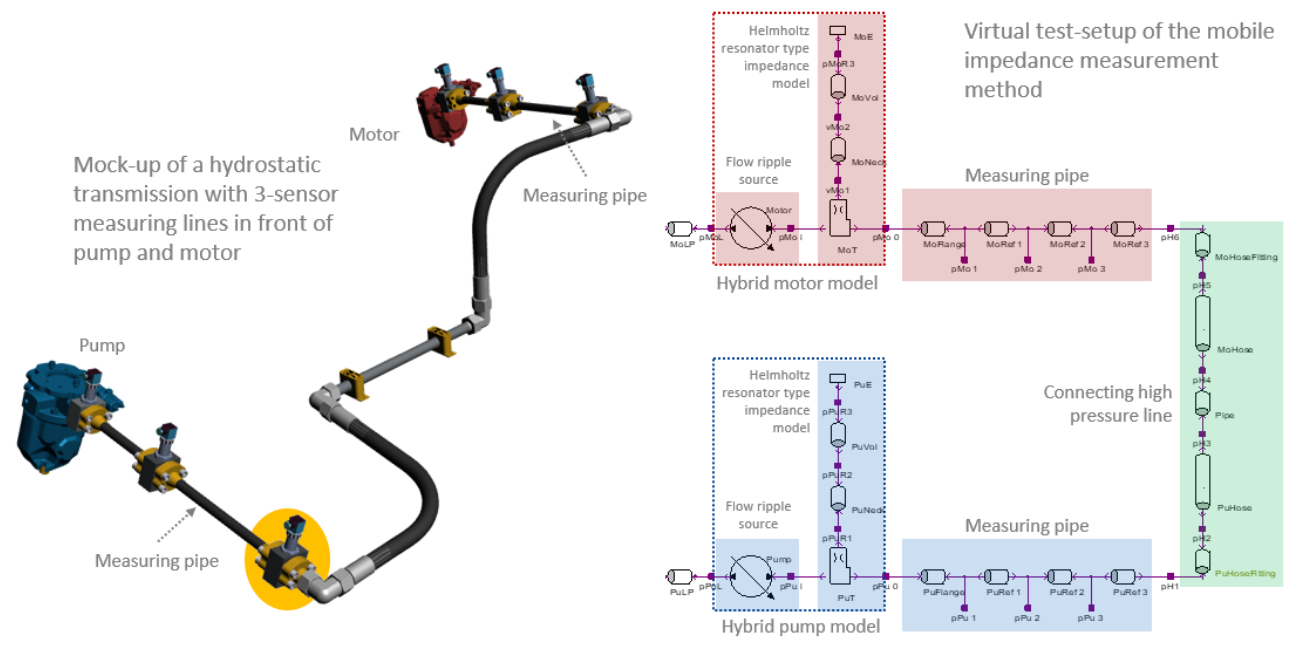

Figure 10: Hydrostatic drive with two reference pipes - simplified simulation model for the virtual development of the "mobile impedance measurement" method 
good approximation, whose parameterization for the virtual test was first estimated.

The test setup developed for "mobile impedance measurement" could also be described as a dual secondary source and $3 \mathrm{p} 2 \mathrm{~s}$ test bench: A reference pipe with the three dynamic pressure sensors and a temperature sensor is installed in the existing piping on the high-pressure side of the pump and motor respectively (see Figure 7 or Figure 8 ). In a first evaluation, the pump serves as secondary source, and the motor's impedance is calculated from the signals of its reference line. In the second evaluation the procedure is reversed, and the pump is the test specimen. However, both evaluations are based on a single runup measurement. The analysis algorithms were developed and tested with "simulated measurement signals". The calculated impedance curves were compared with the theoretical impedance curves.

\section{RESULTS}

In the following the step-by-step procedure of the evaluation and exemplary results are shown.

\subsection{Measurement evaluation and results}

First, the sound velocity in both reference pipes is determined from the measured data. The procedure according to ISO 15086-2:2000 can be applied to the unfiltered data from the run-up without modification. The measured speed of sound serves as input variable in later analysis steps.

Figure 11 shows the results of a total of 35 measurements. The speed of sound is determined for both the pump and the motor side, resulting in 70 data points which are plotted in the 3D diagram as a function of pressure and temperature and which scatter only slightly around the best-fit plane shown in the figure.

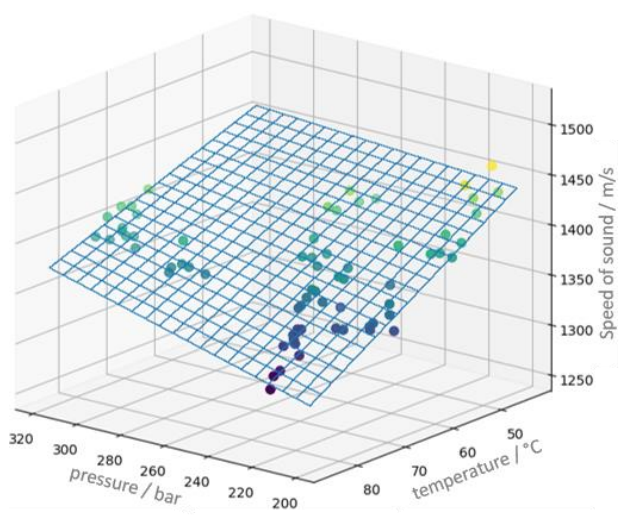

Figure 11: Speed of sound measurement results

The best-fit plane, which correlates well with published curves of pressure- and temperaturedependent speed of sound, makes it clear that large temperature and pressure changes should be avoided in one measurement. If the temperature varies over a series of measurements, further evaluation should take the temperature-dependent speed of sound into account.

In the further sequence (Figure 12), the impedance curve is determined. First, the raw signals are examined in the Campbell diagram, checking that the desired orders can be "separated". If necessary, the transmission ratio of the hydrostatic transmission may be readjusted via the pump and/or motor swivel angle. 


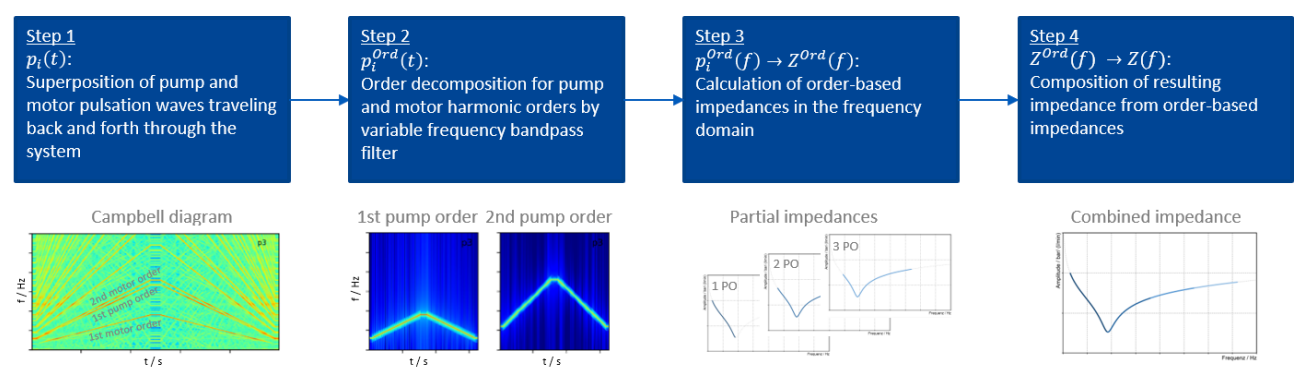

Figure 12: Sequence of the evaluation from the measurement data to the impedance curve

In the second step, the desired orders are filtered out of the time signals of the three specimen-side sensors using a bandpass filter: To determine the motor impedance, for example, the pump orders (pump $=$ secondary source) are extracted from the sensor signals of the motor-side reference pipe. The center frequency of the bandpass filters is calculated using the measured and subsequently smoothed specimen's speed signal.

From the filtered pressure signals, the impedances belonging to each excitation order are determined in the third step. From each usable order an impedance curve results for the frequency range which this order has excited sufficiently (Figure 13 left). This part of the evaluation procedure corresponds approximately to the calculation method proposed in ISO 10767-1:1996. The entire evaluation up to this point is performed semi-automatically via scripts.

Figure 13 on the right schematically visualizes how the "partial impedances" are combined to form the overall impedance, which covers the entire frequency range. Due to the run-up and the resulting continuously varying excitation frequency, not only single points of the impedance are present, but a continuous impedance curve.

An example of resulting impedance curves is shown in Figure 14: The three curves each represent the evaluation for one sensor pair. No excitation takes place in the frequency ranges highlighted in grey, so that no meaningful results are available. Between these ranges, both the amplitude and phase of the three measurement results are almost congruent and indicate upon an attenuation point. In the further course of the measurement, there are somewhat larger deviations in the evaluation of the sensor pair 1-3, whose distance is in the order of half the wavelength, so that the forward and backward travelling waves are partially extinguished, which means that a meaningful
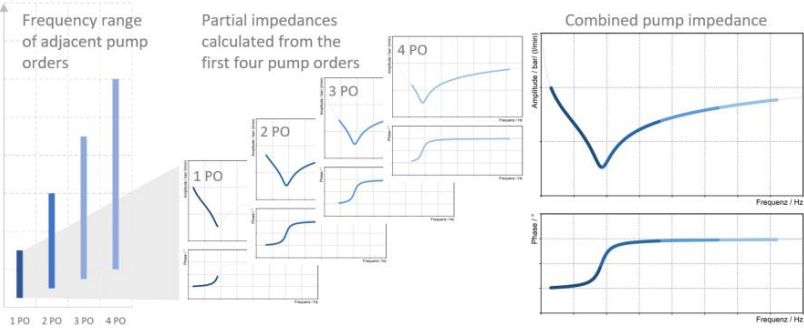

Figure 13: Order-based "partial impedances" and superposition to the overall impedance curve
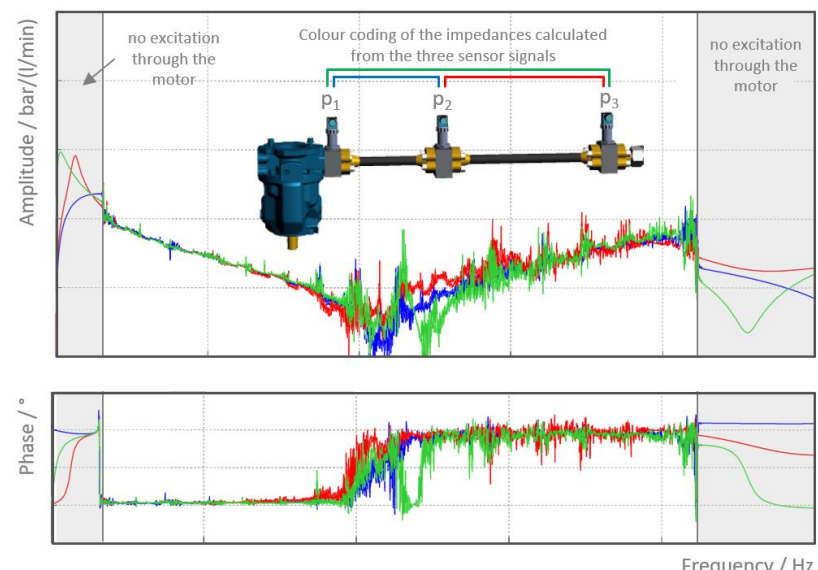

Figure 14: Measured overall impedance curves 

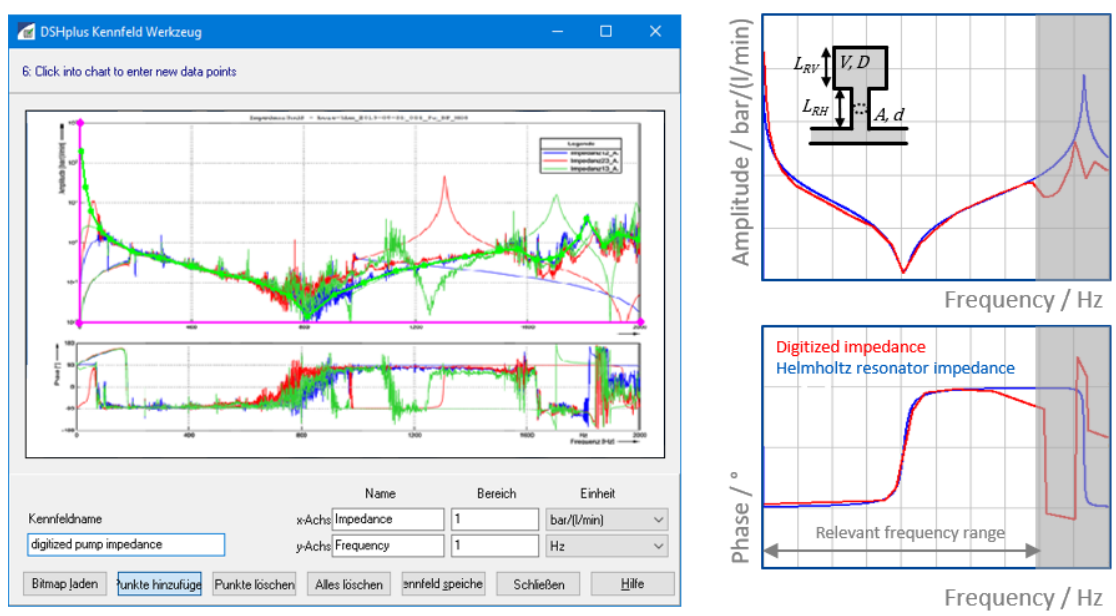

Figure 15: Approximation of measured impedance curves by the analogous model

evaluation is no longer possible. In higher frequency ranges, shorter sensor distances are therefore advantageous, while a larger distance in the low frequency range tends to produce better results. If the reference section is equipped with three sensors, the optimum distance for the frequency range can be selected.

\subsection{Application in hybrid pump model}

In order to make the measurement results usable for 1D simulation in the time domain, they are transferred to the analogous model presented in Figure 5.

First, the measured impedance curves are approximated by an averaged and smoothed curve. Up to now, this has been done visually with the aid of a digitization tool (Figure 15 left). This step can also be automated in the future using suitable averaging methods.

The parameters of the Helmholtz resonator are then adjusted in an automated process with the aid of numerical optimization in such a way that the averaged impedance curves for the frequency range of interest are approximated as closely as possible (Figure 15 right). The correspondingly parameterized analogous model in the time domain then reproduces the impedance characteristics of the displacement unit in good quality with low computing effort.

\section{CONCLUSION AND OUTLOOK}

This paper presents a method for measuring the impedance of displacement units in the hydrostatic circuit. Based on the known methods "secondary source" and " $2 \mathrm{p} 2 \mathrm{~s}$ " the method of "mobile impedance measurement" was derived, which considerably reduces the effort for measuring displacer impedances and is therefore easier to implement in practice. The method offers the following advantages:

- No special test bench is required.

- The impedances of pump and motor are determined in one setup and from one measurement run.

- The measurement data of one measurement run provides impedance curves over a wide frequency range.

- The current speed of sound is calculated from the same measured data.

The exact knowledge of the impedances of the displacers is a prerequisite for the pressure oscillation analysis of the hydrostatic circuit. The presented results were successfully applied for this purpose.

The future goal is to extend the evaluation of the measured data in such a way that the actual flow pulsations are also calculated from the measured data.

\section{REFERENCES}

[1] Kohmäscher T (2008) Modellbildung, Analyse und Auslegung hydrostatischer Antriebsstrangkonzepte. Dissertation, RWTH Aachen 
[2] FLUIDON Gesellschaft für Fluidtechnik $\mathrm{mbH}$ (2017) DSHplus - Simulationsprogramm für fluidtechnisch mechatronische Systeme FLUIDON. Aachen

[3] Baum H (2019) Druckschwingungsanalyse hydostatischer Antriebsstränge. O+P Fluidtechnik 6/2019:36-41

[4] Veit I (2005) Technische Akustik: Grundlagen der physikalischen, physiologischen und Elektroakustik, Auflage: 6 . (Juni 2005). Vogel Business Media, ISBN-13: 978-3834330130

[5] Möser M (2015) Technische Akustik, Auflage: 10 (30. Oktober 2015). Springer Vieweg, ISBN-13: 978-3662477038

[6] Baum H (2016) Druckschwingungsanalyse hydraulischer Leitungssysteme Eine Alternative zu CFD. DIGITAL ENGINEERING Magazin 04-2016:30-32

[7] Baum H, Becker K, Faßbender, A (2014) Hybrid Pump Model for 1D Hydraulic System Simulation. The 9th International Fluid Power Conference, 24. - 26.3.2014, Aachen

[8] BS ISO 10767-1:1996: Hydraulic fluid power - Determination of pressure ripple levels generated in systems and components - Part 1: Precision method for pumps

[9] ISO 10767-1:2015(E): Hydraulic fluid power - Determination of pressure ripple levels generated in systems and components - Part 1: Method for determining source flow ripple and source impedance of pumps

[10] Liu, J, Suh, S, Butts, T (2018) Source Flow Ripple and Source Impedance Measurement for Different Hydraulic Pumps. INTER-NOISE 2018, Chicago

[11] ISO 15086-2:2000(E): Hydraulic fluid power - Determination of the fluid-borne noise characteristics of components and systems - Part 2: Measurement of the speed of sound in a fluid in a pipe

[12] ISO 15086-3:2008(E): Hydraulic fluid power - Determination of the fluid-borne noise characteristics of components and systems - Part 3: Measurement of hydraulic impedance 